\title{
Neurofisiología de la alimentación: su incidencia en la obesidad común (I)
}

\author{
T. Pujol Sanso
}

Universidad de Palma de Mallorca

\section{INTRODUCCION: REVISION CONCEPTUAL}

La alimentación constituye un tema de especial interés en multitud de campos de estudios y por muy distintas razones, recordemos con Fischler (1979), que en la actualidad asistimos a un gran número de corrientes alimenticias, de creencias, sectas, escuelas dietéticas o estéticas; la comida nunca había ocupado un lugar tan importante. En este conglomerado hay de todo y, en general, una gran confusión entre mitos pasados y delirios innovadores.

El objetivo primordial de la toma de alimento es la nutrición, que, de acuerdo con Guerrero (1979), permite a los seres vivos mantener sus funciones, crecer y reparar sus tejidos. Ahora bien, el hecho de alimentarse tiene, por otro lado, connotaciones muy vinculadas al desarrollo psicosocial del ser humano, baste señalar que supone el primer vehículo, o por lo menos el más importante, de comunicación entre el niño y su entorno.

La alimentación supone una encrucijada biológica, psicológica y social, por ello su estudio exige un punto de vista interdisciplinario e integrador. Compartimos la opinión de Bolles (1976, p. 57): «Las aplicaciones especializadas de las ciencias no conducen al reduccionismo, sino a la formación de puentes científicos". Es preciso seguir este enfoque y mantener una actitud decidida para descubrir las falacias y malentendidos que pueblan todo lo que está relacionado con la alimentación.

Estos argumentos son aún más esenciales cuando nos acercamos a las posibles patologías que puede originar una determinada toma alimentaria; la más frecuente, cerca del so por 100 de personas mayores de cuarenta años la padecen (Cradoock, 1969, y Sánchez Ocaña, 1980), y tema de nuestra revisión, es la obesidad común.

El hecho de que la obesidad se mani- 
fieste a través del volumen corporal, con los consiguientes peligros para la salud (Vague, 1972; Gotto, 1976, y Apfelbaum y Lepoutre, 1978), y los problemas psicosociales de todo tipo, de los que podriamos destacar por ahora la vivencia estética frente a una sociedad agresiva ante el exceso de peso, convierten esta alteración en foco de controversia. Los inconvenientes de esta dolencia han dado lugar, en muchas ocasiones, a tratamientos milagrosos con fines puramente lucrativos. De alguna manera, el tratamiento fácil y económicamente rentable ha conducido, y esto es todavía más grave, a dar explicaciones etiológicas falsas o por lo menos tendenciosas. Descubierto un tratamiento conveniente era necesario crear una etiología que lo fundamentara.

Como veremos, en el estado actual de conocimientos no es válido, salvo en casos muy concretos, detallar causas únicas o etiologías simples. El sobrepeso ocurre merced a un cuadro complejo que en muchas ocasiones es dificil descifrar.

Queremos dejar bien claro que nuestro interés por los aspectos psicológicos y el convencimiento de su influencia esencial en la génesis de la obesidad común, no ha entorpecido la revisión y aceptación de otros campos de estudio.

En las líneas anteriores nos hemos referido sucintamente a los conceptos de alimentación y nutrición, queremos hacer lo mismo con otros conceptos de interés para nuestro tema.

El tradicional concepto de "hambre» ha sido definido de múltiples maneras según el contexto teórico en que era utilizado, en general, ha mantenido un sentido de algo intrínseco, no aprendido, con carácter de pulsión o de instinto (Genevard, 1952). Si bien hay que reconocer que el hambre es una característica esencial del ser humano, es preciso comprender que su significado puramente instintivo queda notablemente insignificante frente a la noción de "apetito" o "deseo de alimento", conceptos ambos que rebasan sutilmente al anterior concepto de orden sensorial para transducirlo, siguiendo la terminología de Forgus (1978), a un nivel perceptual e incluso con privilegios importantes de tipo intelectual y afectivo. De la misma manera, la "saciedad" con sus confusas connotaciones fisiológicas debe entenderse muy por encima del nivel sensorial.

La sobrealimentación, pues, tanto en su sentido cuantitativo, exceso de alimentos, como en su sentido cualitativo, consumo demasiado rico en algún elemento básico nutritivo, debe situarse en la misma perspectiva psicobiológica que los conceptos anteriores. Observemos, de acuerdo con Trémolières (1973), que la sobrealimentación carece de sentido cuando se emplea con referencia a una ingestión «normal», ya que se debe referir a cada individuo en particular.

La bulimia es la manera con que se caracteriza una sobrealimentación de carácter psicopatológico (Dongier, I971, y Porot, 1977). La bulimia puede manifestarse de distintas maneras que ya hemos tratado en otro lugar (Pujol, 1980). La aparición de cualquiera de las formas de bulimia dará lugar, en casos determinados, al sobrepeso o su vertiente clínica que es la obesidad común.

La obesidad común ha sido definida en muchas ocasiones, hasta el momento no puede aceptarse ninguna por completo. Esta pluralidad se debe a los distintos puntos de referencia en que se sitúan los investigadores. En conjunto destacan cuatro perspectivas principales:

a) Con referencia a su etiología: aquí podrian incluirse múltiples definiciones atendiendo a las distintas explicaciones etiológicas que existen. De todas maneras, las que se fundamentan en el enfoque termodinámico son las más aceptables. En este sentido Corraze y Bayourthe (1966) indican que la obesidad se explicaría a partir de un desequilibrio entre las 
entradas y salidas; o como define Brengelman (1975, P. I I 3): «La obesidad es el resultado de una sobrealimentación y/o un insuficiente consumo de energian. Señalemos, por último, la definición que dan Zöllner y Keller (1980, p. 19): «La obesidad es la exteriorización de un balance energético positivo a largo plazo; si con la alimentación se suministra más energía de la que pueden transformar el metabolismo basal, el trabajo muscular y otros procesos que consuman, el resto se acumula como grasa en el tejido adiposon.

b) Con referencia a su histología: en esta perspectiva incluimos aquellas definiciones que de una manera u otra se refieren a una acumulación exagerada de grasa. La medición del grado de obesidad desde este punto de vista puede realizarse de dos formas distintas:

- Determinando el espesor del pliegue cutáneo en ciertas partes del cuerpo, con la ayuda de un adipómetro. Existe una obesidad moderada cuando el espesor del piegue cutáneo es mayor a $1,5 \mathrm{~cm}$. en el hombre y $2,0 \mathrm{~cm}$. en la mujer.

- Midiendo el espesor del tejido subcutáneo en condiciones determinadas con ayuda de una placa radiográfica.

c) Con referencia al peso ideal: en este sentido, la obesidad se define como un exceso ponderal en relación al peso ideal. No hay concordancia en cuál es el exceso que define la obesidad, Gordon (1969) admite que un 20 por 100 de exceso la determina, Soulé (196s) considera que debe situarse a partir del is por 100 y Allannic (1973) habla de obesidad media para excesos comprendidos entre el 10 y el 30 por roo y obesidad severa para excesos superiores al 30 por 100.

El problema no termina aquí, tampoco están de acuerdo los autores en cómo calcular el peso ideal. Existen diversas modalidades:
- El índice de Broca es el más sencillo de todos, se calcula a partir de la fórmula siguiente:

Peso ideal $(\mathrm{Kg})=$. Talla $(\mathrm{cm}$. - 100.

- El índice de Broca modificado es el utilizado en un artículo de los laboratorios Sandoz (1975), que se obtiene a partir de la fórmula anterior, pero restándole un ro por 100 a los hombres $y$ un is por 100 a las mujeres.

- La fórmula de Lorentz es también de frecuente uso:

Peso ideal $(\mathrm{Kg})=$. Talla $(\mathrm{cm}$. $-100-($ talla $-150 / 4)$.

- Para el cálcullo del peso ideal existen también diferentes tablas obtenidas con medios distintos y que clasifican los sujetos según talla, edad y, en algunos casos, constitución. La más utilizada èn EE. UU. es la del Metropolitan Life Insurance Co. (1959).

d) Con referencia a su conceptualización patológica: aqui hay gran controversia, muchos autores la definen como una enfermedad, otros como un síntoma. En realidad esta polémica, que a primera vista parece puramente teórica, tiene grandes incidencias a nivel diagnóstico y terapéutico. Entender la obesidad como una enfermedad implica una acción directa contra el sobrepeso, el régimen alimenticio y los tratamientos farmacológicos se convierten en centro de atención. La obesidad como síntoma representa una visión más actual y más acorde con los conocimientos de que disponemos. En este sentido Saltiel (1976) es, quizá, uno de los autores que con mayor empeño ha defendido su carácter sintomático, la obesidad es el resultado de una situación, existe un «contexto obesidad». Se trata de la confrontación de una perspectiva organicista, rígida y univoca, con un enfoque dinámico, flexible y multifactorial. 


\section{EL PROCESO METABOLICO}

El hombre es un animal omnívoro, come de todo. Ahora bien, las células no pueden alimentarse de todos los productos, es necesario transformarlos a tres sustancias básicas llamadas "principios inmediatos». Son los hidratos de carbono, las proteínas y las grasas. Estos elementos mediante la digestión se escinden en otros más sencillos que pueden quedar absorbidos por la pared intestinal. Así los hidratos de carbono se convierten en azúcares elementales, principalmente glucosa, las proteínas en aminoácidos y las grasas, fundamentalmente, en ácidos grasos. Una vez que estos tres elementos atraviesan la pared intestinal, la sangre se encarga de transportarlo por todo el organismo. De esta manera, las células efectúan una serie de operaciones que permiten asimilar y aprovechar estos elementos, estas operaciones se conocen con el nombre de metabolismo (Villee, 1968, y Rodwell Williams, 1973). El metabolismo debe entenderse como el conjunto de operaciones de transformación de los principios inmediatos en los elementos básicos fundamentales para cumplir su destino final, incorporarse a la constitución de las células y proporcionar la energía necesaria.

Ya que el cuerpo sólo puede desempeñar su trabajo por medio de la energía y dado el caso de que todo trabajo toma la forma de producción de calor, la energía puede ser medida en términos de equivalentes calóricos. Esta medida es la caloría. Una kilocaloría es igual a mil calorias y supone la cantidad de calor necesaria para elevar un kilogramo de agua a un grado centígrado (Sánchez Ocaña, 1980).

La aportación energética proviene enteramente de la alimentación. En el cuerpo humano la energía se obtiene de cuatro maneras básicas: química, eléctrica, mecánica y térmica. Las fuentes de energía externa se convierten en glucosa, la cual se quema para producir energía, el agua y el bióxido de carbono son los productos finales de este proceso de oxidación. El metabolismo, en este sentido, sería el proceso de convertir la energia química a otras formas de energía para el trabajo del cuerpo. Hay que tener en cuenta que la retención de unas 6.000 calorias representa alrededor de $\mathrm{I} \mathrm{kg}$. de masa adiposa.

Está claro que el proceso metabólico interviene directa y especialmente en la alimentación, pero no ha podido demostrarse su incidencia real en la obesidad, a lo sumo algunos estudios parecen apuntar la posibilidad de que el sujeto obeso forma la masa adiposa con menos calorías que el no obeso. Ahora bien, este proceso no nos dice nada sobre el cómo, cuándo y porqué comemos (entradas) y consumimos (salidas). La manera de alimentarse, los momentos del día en que lo hacemos y las razones que nos llevan a ello, así como el grado de actividad diaria, emergen por encima del proceso metabólico para situarse en el terreno del comportamiento, que incluye además de los elementos propiamente biológicos otros muchos de índole psicosocial.

\section{EL METABOLISMO BASAL Y SU MEDICION}

Se trata de una medida de la energía producida para el mantenimiento del cuerpo en descanso después de un ayuno de I 2 horas. La proporción metabólica basal es la proporción de la actividad química interna del tejido en reposo. Ciertos tejidos pequeños, pero vitalmente activos como el cerebro, hígado, el tracto gastrointestinal, el corazón y los riñones, constituyen entre todos menos del 5 por roo del peso total del cuerpo, pero contribuyen en un 60 por 100 de los procesos metabólicos basales en su totalidad.

Los gastos base suponen cerca de la mitad de las necesidades energéticas de 
un sujeto con un modo de vida normal. Es lógico, por tanto, atribuir a esta función cierta responsabilidad en la posible formación del sobrepeso. Sin embargo, no es menos cierto que los gastos base están influidos por el peso, disminuyen cuando aumenta el peso del sujeto y esto puede inducir al error importante de aceptar por completo la primera suposición de manera que siempre se pensará que la disminución de los gastos base favorece al obesidad, cuando, en realidad, lo cierto, en la mayoría de casos, es justo lo contrario.

Por otro lado, estos gastos también varian según la edad y el sexo, de forma que cuanto más joven más necesidades energéticas motivadas por el crecimiento y además, las mujeres gastan un s ó 7 por roo menos que los hombres. Pero a pesar de que en mujeres con más edad existe mayor proporción de obesidades no se puede pensar que los gastos base sean los únicos responsables, como veremos existen muchos más factores de riesgo.

\section{LOS GASTOS EN SEMIRREPO- SO}

Hemos visto que cerca de la mitad de los gastos energéticos se deben a los gastos base, los gastos en semirreposo consumen alrededor de una sexta parte del total. Estos gastos comprenden la actividad muscular moderada para mantener el cuerpo en pie o sentado y los efectos térmicos de la digestión que empiezan con los primeros movimientos de la masticación y que duran alrededor de tres horas. Los gastos digestivos dependen de los alimentos absorbidos y de la propia función digestiva y en conjunto suponen un 10 por 100 de las calorias consumidas.

Por de pronto conviene resaltar una consecuencia de lo anterior, una cantidad determinada de comida producirá menos gastos si es consumida de una sola vez. Es preciso tener en cuenta estos aspectos para la comprensión etiológica de la obesidad, pero su incidencia será todavía menor que la otorgada a los gastos base.

\section{ร. LOS GASTOS EN TRABAJO}

Aquí se incluyen aquellos motivados por el esfuerzo muscular, ya sea por las necesidades de la profesión, el deporte, o cualquier otra modalidad. Suponen alrededor del 35 por roo del consumo y junto a los gastos base y los gastos en semirreposo componen la totalidad del gasto energético.

Los gastos en trabajo son en realidad económicos, el esfuerzo muscular no es un gran consumidor de energía. Una profesión ligera como la de oficinista consume de 50 a $100 \mathrm{cal} / \mathrm{h}$. y una intensa como la de minero de $500 \mathrm{a} 600 \mathrm{cal} / \mathrm{h}$. En cuanto a los deportes señalemos que el ciclismo en carretera consume $400 \mathrm{cal} / \mathrm{h}$., $\mathrm{y}$ el tenis en simples cerca de $800 \mathrm{cal} / \mathrm{h}$. Como es lógico, la tensión nerviosa y el entrenamiento varían estos datos.

De todas maneras no hay que menospreciar estos gastos, no olvidemos que la energía total consumida durante 24 horas puede exceder las 6.000 calorías si el individuo está comprometido en una labor fisica fuerte. Hay que aceptar, de acuerdo con Konishi (1965), que la actividad física es un factor importante en la etiología de la obesidad; Johnson (1956), estudiando una muestra de 28 mujeres obesas y 28 no obesas, concluyó que la inactividad es un factor mucho más importante que la sobrealimentación. Lo cierto es que muchas veces es difícil discriminar si la inactividad es producto del sobrepeso o viceversa; sin embargo, y esto es claro, la actividad del ser humano es un comportamiento que incluye muchos aspectos psicosociales. 


\section{LAS CONTRACCIONES GASTRICAS}

Uno de los primeros intentos explicativos fue el que a principios del siglo dio Cannon (citado por Bolles, 1976), que, como defensor de la teoría local de la motivación, afirmó que las sensaciones de hambre tenian sus causas en contracciones estomacales. Diversos autores posteriores, investigando con pacientes a los que se les había extirpado el estómago, o utilizando fístulas estomacales, pudieron observar que la hipótesis de Cannon no era cierta, a pesar de que muchas personas comen con el fin de eliminar estas sensaciones. A idénticas conclusiones han llegado estudios de los hábitos alimenticios de obesos (Schachter, 1968).

\section{EL HAMBRE ESPECIFICA}

Conviene tener en cuenta lo que bastantes estudios han evidenciado con respecto al hambre especifica. Se puede afirmar, como regla general (Morgan, 1973), que si un animal necesita una dieta de una composición determinada debido a un desorden metabólico o a una carencia alimenticia artificialmente provocada, desarrollará una necesidad específica hacia este tipo de alimentos. Por otro lado, el medio interno puede también desajustar las necesidades, así el embarazo y lactancia determinan que la madre necesite mayor cantidad de minerales, especialmente sodio, fósforo y calcio, también más grasas y proteínas, todo ello debido a las demandas del feto; es el único fundamento biológico que podría disculpar los conocidos "caprichos» de las embarazadas. Estos cambios metabólicos llevan consigo los respectivos cambios alimenticios. Por regla general, se afirma que en los seres humanos adultos y en condiciones normales, esta función es improbable, los hábitos alimenticios ad- quiridos habrán borrado las autoselecciones beneficiosas a que nos hemos referido.

Es, por tanto, erróneo suponer que la obesidad común puede originarse a partir de esta perspectiva. Pensamos que, quizá, podria hablarse de «hambre de calorías» en determinados períodos del tratamiento del sujeto obeso, en especial cuando se utilizan dietas de bajo contenido calórico. Nuestras experiencia terapéutica nos permite afirmar que entre la sexta y octava semana, por término medio, se presenta una necesidad acuciante de calorias.

\section{FACTORES GUSTAFIVÓS}

Es indudable que el gusto influye en la ingesta e incluso en el hambre específica. Algunas sustancias saben mejor que otras, o se prefieren cuando el cuerpo tiene mayor necesidad de ellas. Diversas experiencias muestran que el factor oral interviene también en la saciedad o mejor en la cesación de la alimentación. Ahora bien, el máximo rango que puede ocupar el factor gustativo es de "facilitador», en ningún caso por sí mismo podrá originar un sobrepeso, su función principal es discriminativa.

\section{EL ARCO REFLEJO ALIMENTARIO}

Bajo esta afortunada expresión de Creff y Herschberg (1979), se agrupa un proceso complejo que veremos a continuación y cuyo. soporte teórico es de orden neurofisiológico. Supone un elemento de especial interés para la comprensión del proceso alimenticio.

\section{Las vías de información}

Las necesidades del organismo se generan en los adipocitos, que constituyen la masa grasa. Estas informaciones llegan a los centros a través de las vías metabólica y nerviosa. 
- La via metabólica: Ya nos hemos referido antes a la hipótesis de las contracciones gástricas, que se asociaron después a la hipoglucemia, la hiperglucemia cesaba las contracciones. Desde 1966 se ha cambiado la explicación, no se trata solamente del nivel absoluto de glucosa en sangre, sino la disponibilidad de la glucosa por las células, en particular de las cerebrales. El índice de utilización celular de glucosa en sangre arterial y la de sangre venosa de un mismo territorio, se representa por ( $\Delta$-glucosa); cuando el índice está próximo a cero aparece el hambre y desaparece cuando la diferencia es de is a $20 \mathrm{mg}$. por $\mathrm{ml}$. Existirán para ello una células sensibles en el S.N.C. llamadas "glucorreceptores", aunque todavía no se han podido identificar.

Los ácidos grasos libres, las aminas y el alcohol parecen jugar respectivamente un papel importante, sin embargo, los mecanismos no son conocidos por completo.

- La via nerviosa: Las informaciones que dan las vías metabólicas son lentas, la más rápida es la de glucosa que de todas meneras dura cerca de media hora. Es preciso, pues, que los centros utilicen unos medios de información más rápidos que indiquen el nivel de saciedad. Estas informaciones son de orden sensorial y se consiguen después de un aprendizaje.

Se puede llegar a controlar la alimentación a partir de la acción de la memoria que registra las características de la comida que se va a consumir. El olor, sabor, consistencia, apariencia, volumen, etc., al ser comparado con los consumos anteriores permite dar unos límites de saciedad o mejor -de consumo. De esta manera participan diversos sentidos:

a) El gusto, que transmite a los centros nerviosos los estímulos que discrimina.

b) El olfato, que estimulado por las moléculas volátiles también ofrece información. c) El papel del estómago; que mediante la distensión juega un papel importante.

Resulta, pues, que las cualidades olfato-gustativas de un alimento, asociadas a los componentes visuales, auditivos y táctiles, junto, además, a los componentes interoceptivos, constituyen esta segunda vía de información.

- Interrelaciones entre la via metabólica y nerviosa: La ingestión alimenticia se caracteriza por la frecuencia (vía metabólica) y la cantidad (vía nerviosa, sensorial). En un sujeto normal los informes metabólicos y los estímulos sensitivo-sensoriales están equilibrados, en el sujeto obeso parece suceder que es más sensible a los estímulos externos que a los metabólicos.

\section{Los centros reguladores}

De acuerdo con Aimez (1972), podemos diferenciar los siguientes puntos:

a) La existencia de un "feeding center» (centro regulador de la alimentación) hipotalámico, que está bien comprobado. Comporta un centro de la saciedad repre sentado por los núcleos ventromediales y ciertas áreas laterales que activan el hambre.

b) Las estructuras vecinas parece que están estrechamente correlacionadas con los centros del hambre y la saciedad, en especial, los centros del sueño y la.vigilia y las estructuras reguladoras del comportamiento agresivo y sexual.

c) El rinencéfalo (o sistema límbico), que actúa inhibiendo el hipotálamo.

d) Las estructuras neocorticales que parecen activar el hipotálamo.

En conjunto puede decirse que una vez que el metabolismo celular llega a un punto crítico, el hipotálamo informado por los estímulos metabólicos induce al comportamiento de tomar comida.

La ingestión de comida pone en marcha los mecanismos sensoriales de la saciedad. Una vez que los nutrientes han 
franqueado la barrera digestiva, los estímulos metabólicos mantienen la saciedad. La duración de la comida y su calidad serán los responsables del aporte energético real. Desde luego que en la realidad es algo más complicado, ya que existe una interrelación constante.

\section{Las respuestas}

Aquí podríamos incluir las distintas conductas alimenticias que pueden darse para cada sujeto (Pujol, 1980).

\section{De la rata al ser humano}

Se han conseguido «obesidades hipotalámicas" en experimentos con ratas por destrucción de los centros ventromediales. El sobrepeso evoluciona en dos fases bien caracterizadas:

1. ${ }^{a}$ Dinámica, de intensa hiperfagia con ascensión ponderal.

2. ${ }^{a}$ Estática, el animal llega a un peso estable elevado y el comportamiento alimenticio se ajusta a un nuevo nivel.

El animal presenta además trastornos del comportamiento motor (por defecto o por exceso), y del comportamiento agresivo y sexual. Además siente un fuerte rechazo a esforzarse para conseguir alimento y una sensibilidad exagerada a los estímulos psicosensoriales: apetito discriminativo (come más si la comida es apetitosa y menos si es aversiva).

Debemos preguntarnos si lo expuesto hasta ahora es aplicable al ser humano. Schachter y Rodin (citado por Garrido, 1979), señalan la enorme similitud que existe entre las ratas con lesión y los obesos al compararlos a ratas y personas normales:

a) El obeso come más cantidad de alimentos cuando tienen buen sabor que los normales, y lo contrario cuando tienen mal sabor. b) Por lo general, el obeso come más que el normal, aunque realiza menor número de comidas.

c) El obeso come más rápido.

d) Los obesos son mucho menos activos.

e) Cuando la obtención del alimento requiere esfuerzo el obeso come menos.

f) Las situaciones emocionales determinan mayor ingesta en los obesos, cosa que ocurre en las ratas lesionadas que se caracterizan por su hipermotilidad, excitabilidad y agresividad.

En este mismo sentido opina Klotz (1972), que se refiere a las investigaciones de Stunkart y Mayer, que según él, constatan que bastantes obesos tienen un trastorno funcional hipotalámico que es responsable en parte de su obesidad. Este trastorno puede existir desde el nacimiento, como manifestarse más tardíamente con un choque afectivo o un episodio fisiológico como la pubertad o el embarazo.

Con mayor detalle ya se habían referido Gennes y Buge (1952) al importante papel que el S.N.C. puede jugar en el tema que nos ocupa. Dos tipos de argumentos les permiten afirmar que una perturbación nerviosa es suficiente para provocar una obesidad, uno es de tipo de experimental y el otro clínico. El experimental se basa en los estudios sobre lesiones hipotalámicas; el clínico se refiere a la aparición de obesidades en el curso de ciertas afecciones bien conocidas del S.N.C., este tipo de lesiones son de naturaleza diversa, pero siempre se localizan en el hipotálamo (tumores, infecciones, traumatismos, etc.).

En contraste con lo anterior, Warner y Balagura (1975) piensan que no es posible aplicar las conclusiones de las ratas a los seres humanos, sobre todo en lo que se refiere a la fase estática. Por otro lado, Thompson (1973), desde una perspectiva puramente fisiológica y experimental, indica la necesidad de dar un giro en la 
investigación y abandonar los experimentos con lesión.

Un enfoque radicalmente organicista no es sostenible en la actualidad, y cuando así pudiera ocurrir, ya no estamos frente a una obesidad común, sino ante una obesidad especifica que puede cursar dentro y a consecuencia de una patología determinada.

Revisemos la advertencia que hace $\mathrm{Ba}$ llús (1977, p. 227): «El hombre es un animal en el que lo biológico y lo genético vienen de continuo modulados y organizados por su medio ambiente y el hipotálamo juega un papel primordial en estas posibilidades de integración entre lo interno y lo externom. Más adelante, y refiriéndose concretamente a nuestro te$\mathrm{ma}$, indica que estas afirmaciones se refieren a un nivel experimental, y por lo tanto, este contexto elimina una amplisi$\mathrm{ma}$ gama de factores biológicos y de ambiente. Dice textualmente luego: "Quiere ello decir que el proceso psicofisiológico del hambre en el hombre o en el animal superior implica algo más, supera esquematismos puramente fisiológicos y entra dentro del capítulo de aquellas conductas complejas en que a las pautas primarias de comportamiento se añaden otros factores determinados de aprendizaje, motivacionales y de situación ambiental y aun socioculturales en el caso del hombre».

Está claro, "el arco reflejo alimentario» supone un aspecto estructural de especial interés, es el medio en donde podrá ocurrir una disfunción operada desde la perspectiva psicosocial.

Ya hemos indicado que las obesidades de orden lesional no pueden incluirse en el marco de la obesidad común. Incluso la vía nerviosa de información revisada antes, debe ser entendida no como una vía sensorial sino perceptiva, con todos los atributos a los que nos hemos referido en la introducción de este artículo.

En cuanto a las respuestas o conductas alimenticias es obvio que no dependen únicamente de las vías de información y de los centros reguladores, ciertos hábitos y aprendizajes ocurridos a lo largo de la vida tendrán. un peso específico de notable importancia. Además, está claro que el córtex está influido por mecanismos psicológicos capaces de alterar el circuito de la alimentación.

\section{APORTACIONES DE LA FARMACOLOGIA}

Se admite que los mecanismos de la sociedad son preferentemente adrenérgicos, mientras que el hambre depende de los colinérgicos; las anfetaminas, los inhibidores de la M.A.O., la L-Dopa y la reserpina potencializan por mecanismos diferentes las tasas de catecolamias cerebrales provocando una reducción de la toma alimentaria.

Por otro lado, la aplicación directa bilateral de una sustancia alfabloqueante al nivel de las sinapsis terminales de las "fibras de la saciedad», lleva una intensa hiperfagia en la rata, comparable a la que provoca la destrucción del núcleo ventromedial.

Este resultado sería debido a la saturación de los receptores betaadrenérgicos por el exceso del nuevo mediador creado por el bloqueo de los alfa receptores. Este juego entre receptores alfa y betaadrenérgicos constituye quizá la clave del refuerzo de los estímulos psicosensoriales por los estímulos metabólicos. Aquí cabe la misma valoración que hemos hecho para las investigaciones con lesión.

\section{I. LA VERSION ENDOCRINOLOGICA}

De siempre la obesidad ha venido asociada a la endocrinología, conceptos tan dispares como la regulación hormonal del 
hambre (Marañón, 1972), o la negación absoluta de la influencia de las hormonas en la etiopatogenia de la obesidad (Saltiel, 1973; Allannic, 1975), han sido mantenidos en las últimas décadas. En la actualidad son muchos los pacientes e incluso médicos que reclaman a todas horas el examen de sus glándulas o la de sus pacientes. En estos momentos la endocrinologia distingue entre aquellas afecciones con sustrato hormonal evidente y que cursan con obesidad, de aquellas en las cuales los estudios más sofisticados de laboratorio no permiten objetivar ninguna alteración hormonal o metabólica. Veamos ciertas perspectivas descalificadas ya en la actualidad:

- La insuficiencia tiroidea: $\mathrm{Si}$ bien es cierto que la insuficiencia tiroidea puede cursar con obesidad, está claro que esto no ocurre en la mayoria de casos. La sola medida del metabolismo basal ha llevado muchas veces a la prescripción de una opoterapia tiroidea a dosis suprafisiológicas. Sus efectos a corto plazo son siempre satisfactorios por su acción catabólica difusa, pero no solucionạn jamás la obesidad; en general, se da el abandono del tratamiento que se decide por el mismo sujeto ante la aparición de taquicardia o de un estado de excitación psicológica.

- La insuficiencia bipotalámo-hipofisaria-gonadal: Que constituye lo que se conoce como síndrome adiposo-genital y que se presenta justo antes de la pubertad con obesidad, retraso del desarrollo genital, etc. Esta alteración ha llevado a tratamientos tan diversos como la administración de gonadotrofinas hipofisarias y/o sustancias androgenizantes. En la actualidad no se puede sostener, desde un punto de vista científico, un fallo hipotálamohipofisario ya que las mediciones hormonales anterohipofisarias no muestran alteración o déficit alguno.

- La obesidad por retención bidrica: Esta perspectiva merece aún mención, en la actualidad siguen aplicándose sustancias diuréticas para el tratamiento de la obesidad. Está claro que pueden encontrarse personas con una retención hídrica importante, pero nunca deberá interpretarse como obesidad. No olvidemos que los diuréticos son eficaces a corto plazo, ineficaces a plazo medio y peligrosos a largo plazo (hipotensión arterial, pérdida de potasio, etc.).

En definitiva, en la actualidad los trastornos endocrinos asociados a la obesidad están bien pefilados y suponen entidades bien caracterizadas. Desde luego, como indica Klotz (1972), es posible que existan disfunciones neurometabólicas que hoy en día no conocemos, asi por ejemplo, son diferentes las obesidades según el factor individual de utilización de los alimentos por la lipogénesis.

\section{2. EL TEJIDO ADIPOSO}

Muchas investigaciones se han dirigido hacia la fisiología del tejido adiposo para poder explicar la obesidad. En realidad, el peso y el volumen del tejido graso es el medio con que se expresa el exceso ponderal. Así Creff y Herschberg (1979), señalan que un hombre normal de $70 \mathrm{Kg}$. tiene is $\mathrm{Kg}$. de grasa, $6 \mathrm{Kg}$. de proteínas y 300 gramos de glúcidos; que corresponden respectivamente a $135.000,24.000$ y 1.200 calorias. En cambio, en un sujeto obeso de $100 \mathrm{Kg}$., se encuentran $40 \mathrm{Kg}$. de grasa (360.000 calorías), $7 \mathrm{Kg}$. de proteínas (28.000 calorías) y 300 gramos de glúcidos (1.200 calorias). Se puede decir que en el obeso la masa de grasa representa el 90 por roo de la energía almacenada.

El tejido adiposo juega un papel importante como aislante térmico, desde el punto de vista mecánico sirve de colchón amortiguador y lubrificante, por otra parte, constituye un lugar de almacenamiento de energía. La capacidad de almacenar energía bajo la forma de grasa representa 
una condición indispensable para la supervivencia.

La capacidad metabólica particular de la célula adiposa consiste en la posibilidad de:

a) Poner en reserva energía bajo la forma de triglicéridos (grasa neutra) con bajo contenido de agua (Is por I00) y con gran capacidad de reserva (alrededor de 7 calorias/gr.).

b) Suministrar energía al organismo en caso de necesidad por la vía sanguínea bajo la forma de ácidos grasos libres, que cubren el 70 por 100 en cifras redondas de las necesidades totales de energía.

Toda esta actividad viene mediatizada por el especial papel que desempeña la insulina como hormona de almacenamiento, que ejerce a la vez una acción lipogénica y antilipolítica; por otra parte, aumenta la permeabilidad de la membrana de la célula adiposa para la glucosa.

Volviendo pues al posible rol que pueda desempeñar la masa grasa, parece ser que el número de células podría tener una acción importante en la constitución de la obesidad. De esta manera habria que diferenciar entre la hipertrofia y la hiperplasia:

- Por hipertrofia se entiende el aumento de tamaño de las células adiposas; esta obesidad hipertrófica es la que aparece después de la adolescencia por desórdenes en el balance energético, el depośito de grasa es desproporcionado. Tiene buen pronóstico para el tratamiento.

- Por hiperplasia se entiende la multiplicación del número de células adiposas; esta obesidad hiperplásica se suele considerar hereditaria o formada durante la primera infancia y crea condiciones especialmente favorables para la sobrecarga ponderal. En muchas ocasiones el depósito de grasa de las células es proporcionado y el volumen corporal es debido a su número excesivo. En cuanto a su pronóstico es relativamente malo, el número de adipocitos es imposible de dis- minuir y, en el caso de querer disminuir el peso de los mismos, no hay que olvidar que por debajo de un cierto tamaño de los mismos aparece un hambre muy intensa.

En esta perspectiva y aún más reduccionista se expresa Nisbett (citado por Yates, 1977).

Desde luego estas aplicaciones son muy sugerentes, sin embargo, dejan sin explicar muchos aspectos. Saltiel (1973) piensa que afirmar un pronóstico a largo plazo sobre datos histológicos es bastante aventurado. Zöllner y Keller (1980) en artículos muy recientes insisten en que la hipótesis del número de células con predisposición a la grasa todavía no ha podido ser demostrado por completo.

\section{LA PERSPECTIVA GENETICA}

Es indudable que el factor genético debe tenerse en cuenta, y en este sentido son numerosos los autores que se refieren a su influencia, eso sí, cada cual le otorga un determinado peso específico. Así Genevart (1952), después de valorar largamente diversos aspectos psicoanalíticos, incide en que no debe descartarse la predisposición familiar. Gennes (1975), situado en la vertiente organicista, reconoce la escasa compresión que existe en la etiología de la obesidad, si bien afirma que en patología comparada existen múltiples ejemplos que demuestran que algunas obesidades son de origen genético a través de caminos metabólicos.

Las estadísticas demuestran gran incidencia de sobrepeso en los hijos de obesos: un is por roo tiene el padre y la madre del peso normal. Un 30 por 100 tienen el padre o la madre obesa y un $4{ }^{\circ}$ por 100 tienen el padre y la madre obesos.

Uno de los autores que con mayor vigor ha defendido el factor hereditario es Astwood (1962), que, basándose en la 
pobreza de los tratamientos existentes, propone que la obesidad es un defecto determinado genéticamente y las personas que así han nacido no se les puede cambiar. Al contrario, Saltiel (1973) afirma, entre otras cosas, que el factor genético es muy discutible, pues resulta que el peso al nacer no condiciona jamás el peso del adulto, además es muy difícil diferenciar el hipotético factor genético.

$\mathrm{Al}$ admitir la etiología genética hay que preguntarse por las vías orgánicas a través de las cuales se podrá transmitir el trastorno. Se acepta, en general, además de los caminos metabólicos, las alteraciones de los centros del hambre o la saciedad, o debido al número de adipocitos que se tenga al nacer. Es indudable que estas explicaciones son pocos fiables y ya nos hemos referido a ello en los apartados correspondientes.

En definitiva, creemos que es justo reconocer que en los últimos años este posible factor hereditario ha sido poco estudiado, pero está claro que en estos momentos sólo puede aceptarse la noción de «predisposición familiar». El mayor inconveniente de los estudios estadísticos es que resulta muy difícil poder afirmar si la herencia es biológica o ambiental.

\section{CONCLUSION}

El amplio abanico de posibilidades que hemos repasado hasta ahora, y que de alguna manera se relacionan directa o indirectamente con procesos neurofisiológicos, nos ofrecen una buena visión del sustrato orgánico de la obesidad, pero no consiguen explicar por completo su compleja dinámica etiológica.

En el ser humano es poco menos que imposible descubrir procesos de tipo conductual, y la alimentación lo es, que no estén sometidos a la influencia psicosocial.

\section{Referencias}

Aimez, P.: «Controle du comportément alimentaire, role de l'environement dans l'obésité humaine». Revue de Praticien, 1972, 6, 805-821.

AlitanNic, H.: «Examen et traitement d'un obèse en pratique médicale courante». Concours Médicale, 1975 , 95, 7.053-7.059.

Apfelbaum, M., y LePoutre, R.: Les mangeurs inegaux. París: Stock, 1978.

As'Tw(X)D, F. B.: The heritage of corpulence. Endocrinology, $1962,71,337-341$.

Balu.us, C.: Compendio de psicofisiologia. Barcelona: Círculo Ed. Universo, 1977.

Bot.les, R. C.: Teoria de la motivación. México: Trillas, 1976.

Brengei.man, ]. C.: "Terapia de dependencias y adicción: tabaco, comida, alcohol y drogas". Análisis y Modificación de Conducta, $1975,0,105+127$.

Corraze, J., y Bayourthe, L.: «Le problème psychologique dans le traiment de l'obésité commune». Concours Médical, 1966, 88, 4.039-4.042.

CRADDOCK, S.: "Obesity and its management». Edinbur and London: Livingstone I.. T. D., 1969.

Creff, A. F., y Herschberg, A. D.: Abrége d'obésité. Paris: Masson, 1979.

DONGIER, M.: Neurosis y trastornos psicosomáticos. Madrid: Guadarrama, 1971 .

Fischlek, C.: «Alimentación infantil e infantilización de la alimentación». Infancia y Aprendizaje, 1979, 8, 57-64.

Forgus, R. H.: Percepción, proceso básico en el desarrollo cognoscitivo. México: Trillas, 1978.

GARrido, I.: «Alteraciones de la conducta alimentaria en humanos». Revista de Psicologia General y Aplicada, $1979,159,661-671$.

Genevard, G.: Aspects psychiatriques et psychologiques de l'obésite. Praxis, 195 2, 4I, 393-412.

GENNES, J. L.: «Les trobles alimentaires des adolescents». Revue de Neuropsychiatre Infantile, i 975, 23, 405-424.

GENNES, J. L., y BuGE, A.: "Les obésités d'origene nerveuse". Revue de praticien, 1952, 16, $1071-1121$.

GORDON, E. S.: «The present concept of obesity». Medical Times, 1969, 97, 142-1 59.

Gorto, A. M.: “Cardiovascular risk parameters". Fn B. J. Will.lAws (Fd.): Obesity. Beharioral Approaches to dietary management. New York: Brunner/Mazel, 1976.

Guerrero, J.: «La alimentación infantil». En Nacer y Crecer. Madrid: Orgaz, 1979.

JOHNSON, M. L.: «Relative importance of inactivity and evereating in the energy balance of obese high school girls". American Journal of Clinical Nutrition, 19,6, 4, 37-44. 
KlotZ, H. P.: «L'obésité problemes psychologiques et psychosomatiques». Gaq. Med. Fr., 1972, 79, $3.831-3.834$.

Konishi, F.: «Food energy equivalents of various activities». J. Am. Diet. Ass., 1969, 46, 186-188.

LEON, G. R.: «Emotional arousal, eating patterns and body image as differetial factors associated with varying success in maintaining a weight lossm. Journal of Consulting and Clinical Psychology, 1973, 3, 474-480.

MaRaNion, G.: "Gordos y flacos». Obras completas, tomo VIII. Madrid: Espasa-Calpe, i 972.

Metropolitan Life Insurance Co.: «Build and blood pressure study, 1959". Soc. of Actuaries. Chicago, 1959.

Morgan, C. T.: Psicologia fisiológica. Madrid: Del Castillo, 1973.

POROT, A.: Diccionario de psiquiatria. Tomo I. Barcelona: Labor, 1977.

P(jol, T.: «Caractereologia del obeso». Mayurqa (en prensa), t980.

RoDWell. Williams, S.: Nutrición y dietoterapia. México: Pax México, 1973.

S.ALtiel, H.: «L'Obésite-Maladie: Un faux concept». Sem. Hôp. Paris, I973, so, 3.377-3.381.

SALTIEL, H.: «L'obésité-symntôme: Classification psychoidynamique et conduite thérapeutique». Sem Hôp. Paris, $1974,53,3.629-3.638$.

SALTIEL, H.: «Conduite a tenir devant une obésité». Revue de Medecin, 1976, 39, 2.158-2.163.

SANCHEZ OCANA, R.: "Obesidad". En Más vale prevenir, n. ${ }^{\circ} 4$. Madrid, I980.

SAXDOZ LABORATORIOS: Exceso de peso. Métodos para su reductión. Madrid: Sandoz, 1975.

SCHACHTER, S.: "Effects of fear, food deprivation, and obesity on eating". Journal of Personality and Social Psichology, 1968, 2, 91-97.

SOLLE, M.: "L'obésité chez l'enfant. Aspects psychosomatiques». Cabierd Med. Lyonnais, 1969, 4I, 1. I 23-1. 128.

THOMPSON, R. F.: Fundamentos de psicologia fisiologica. México: Trillas, 1973.

Tremolieres, J.: «Obesity as a global problemm. Proc. Nutr. Soc., 1973, 32, $169-174$.

VAGLE, J.: «L'Obésité: Aspects métaboliques, psychologiques-thérapeutiques». Rev. Lyonnais Mediter, 1972, $28,2.801-2.832$.

Vil.1.EE, C. A.: Biologia. México: Interamericano, 1968.

WARNER, K. E., y BALAGLiRA, S.: «Intrameal eating patterns of obese and nonobese humans». Journal of Comparative and Phisiological Psycbology, 1975, 7, 778-783.

Y.iTF.s, A. J.: Terapia del comportamiento. México: Trillas, 1977.

ZOLI.NER, N., y KELLER, C.: "Tractament de l'adipositat: Dieta zero o dieta reduida en calories? Dieta reduida en caloriesm. L'Internista, 1980, 1, 19-28.

\section{Resumen}

En este estudio revisamos y valoramos las distintas explicaciones etiológicas de la obesidad comun, que de alguna manera se fundamentan en principios neurofisiologicos. Entre otras, se estudian las influencias del proceso metabólico, del tejido adiposo, de la berencia genética $y$, en especial, del "arco reflejo alimentario». Se evidencia la limitación que supone una perspectiva radicalmente organicista para la comprensión de la obesidad, que en la inmensa mayoria de casos necesita del soporte psicosocial.

\section{Summary}

In this study, we revise and valuate the different etiological explanations of normal obesity, which in some way is based on neurophysiological origins. Among otber, the influences of the metabolic structure, of the adipose tissue, of genetic inbetitance, and especially of the "ralimentary reflex arc" are studied. It's evidenced the limitation that assumes a perspective radically organicist of the under standing of obesity which in the grand majority of cases needs psycosocial support.

\section{Résumé}

Dans cette étude, on passe en revue et on évalue les différentes explications étiologiques de l'obésité commune, qui d'une certaine fagon se basent sur des principes neurophysiologiques. Entre autres, on étude les influences du processus métabolique, du tissu adipeux, de l'béritage génétique et surtout, de "l'arc réflexe alimentaire». On met en évidence la limitation qui suppose une perspective radicalement organiciste pour la comprébension de l'obésité, qui dans linmense majorité des cas a besoin d'une base psycbosociale. 